\title{
Column-Based Graph Layouts
}

\author{
Gregor Betz, Christoph Doll, Andreas Gemsa ${ }^{\star}$, \\ Ignaz Rutter, and Dorothea Wagner \\ Karlsruhe Institute of Technology (KIT) \\ firstname.lastname@kit.edu
}

\begin{abstract}
We consider orthogonal upward drawings of directed acyclic graphs (DAGs) with nodes of uniform width but node-specific height. One way to draw such graphs is to use a layering technique as provided by the Sugiyama framework [10]. However, to avoid drawbacks of the Sugiyama framework we use the layer-free upward crossing minimization algorithm suggested by Chimani et al. and integrate it into the topology-shape-metric (TSM) framework introduced by Tamassia [11. This in combination with an algorithm by Biedl and Kant 2] lets us generate column-based layouts, i.e., layouts where the plane is divided into uniform-width columns and every node is assigned to a column.

We show that our column-based approach allows to generate visually appealing, compact layouts with few edge crossing and at most four bends per edge. Furthermore, the resulting layouts exhibit a high degree of symmetry and implicitly support edge bundling. We justify our approach by an experimental evaluation based on real-world examples.
\end{abstract}

\section{Introduction}

One of the most well-known approaches for drawing directed acyclic graphs (DAGs) is the Sugiyama framework [10. It consists of three steps, each of which can be solved individually: (i) layer assignment, (ii) determining relative positions within each layer to reduce edge crossings and (iii) positioning the vertices and edges. In the first step the nodes are assigned to layers such that the target of each edge is in a layer below its source. The second step reduces the number of edge crossings by changing the order of the nodes within each layer. Finally, coordinates are assigned to the nodes as well as to bend-points of the edges.

The Sugiyama framework suffers mainly from two drawbacks. First, computing an unfortunate layering of the nodes in step (i) can enforce crossings in step (ii) which would not be necessary if another layering was chosen. Second, due to the way the layers are defined a single node with large height can lead to non-compact layouts (see Figure 1(a). One approach that resolves the first problem but still leaves the second is the layer-free upward crossing minimization approach by Chimani et al. [3], which was also integrated into the Sugiyama framework [4].

* Financially supported by the Concept for the Future of KIT within the framework of the German Excellence Initiative. 
Motivated by the application Argunet (www.argunet.org) that is used to display the structure of debates or argumentational analyses, we focus on orthogonal upward drawings of DAGs whose nodes are represented as rectangular boxes of uniform width. Graph drawings with given node sizes has been explored by Di Battista et al. [5] they consider planar graphs with a fixed embedding and arbitrary node sizes. We deal with non-planar graphs without a given topology but with the restriction that the nodes have uniform width. For minimizing the number of crossings we use the algorithm by Chimani et al. [4 but, instead of the Sugyiama framework, we integrate it into topology-shape-metrics framework (TSM) invented by Tamassia [11. The TSM-framework can be seen as a three-phase-method for orthogonal graph drawing: (i) fixing a planar embedding, (ii) computing an orthogonal description and (iii) compaction of the layout.

The integration of the approach by Chimani et al. with the TSM-framework lets us construct column-based graph layouts. In a column-based layout the plane is divided into disjoint columns of uniform width that correspond to the uniform width of the boxes. The boxes and edges are then assigned to these columns. Thereby, vertical edge segments always run within a column, whereas horizontal edge segments may span several columns. Due to the columns the final layouts have a clear look, which is exemplified in Figure 1(b). The same graph with a layer-based layout is shown in Figure 1(a).

We consider the following drawing style for our graph layouts: The drawings we generate are orthogonal upward drawings of DAGs whose nodes are represented by boxes of uniform width, and individually prescribed heights. We require that edges leave their sources at the bottom and enter their targets at the top. We also include minimum spacing constraints that ensure that there is a minimum space between two boxes, a box and an edge and two parallel edge segments. We allow to specify two different spacing constraints for the case of two parallel edge segments: one for distance between two edge segments whose edges have a common source or target and one for the remaining cases. By using these two different minimum spacings we enable edge bundling.

Besides the mentioned drawing style, our approach guarantees certain properties that are aimed at creating visually appealing drawings. When looking at a single box of the layout, it is important that its predecessors can be found quickly. In order to give them a unifying look, we want as many predecessors as possible of each box to be vertically aligned at their bottom. Additionally, we show how to assign nodes into columns such that for each node $v$ its assigned column is the median of the columns assigned to the incoming edges of $v$ as well as the median of its outgoing edges. We call this property local symmetry. Finally, we guarantee that the edges will have at most four bends.

While satisfying these properties, we optimize the following three criteria: (i) number of edge crossings, (ii) number of bends and (iii) total edge length. These optimization goals are frequently used when drawing graphs [7.

Contribution and Outline. Our main result is an algorithmic approach to generate column-based graph layouts for directed acyclic graphs. This approach is based on the topology-shape-metric framework and utilizes Chimani et al.'s 


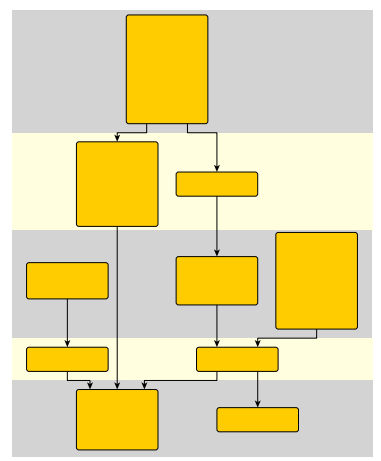

(a) A layer-based layout (computed by yEd).

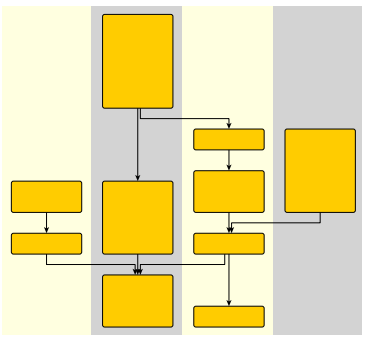

(b) A column-based layout (our approach).

Fig. 1. A column-based and a layer-based layout of the same graph

layer-free upward crossing minimization. Contrary to the standard approach of the TSM-framework, we do not fix the topology computed in the first step but use it only as a basis for computing a column-assignment of the nodes in the second step. In the last step we only use this assignment and ignore the topology from the first step. This lets us focus on optimizing other criteria than the number of edge crossings.

Note that we use the "upward" terminology throughout this paper, because it is established in the graph drawing community. Nevertheless, since this drawing style originates from a particular application - which requires that all edges are directed downwards - all figures in this work have downward directed edges.

The remainder of the paper is structured as follows. In Section 2 we give a detailed description of the algorithmic approaches we use in every step of the TSM framework. We give an experimental evaluation of our approach in Section 3. We omit several details of our proofs due to space constraints; full proofs can be found in the appendix.

\section{Topology-Shape-Metrics}

The TSM framework consists of the three basic steps topology, shape and metrics. The key idea is that reducing the number of crossings is the most important æsthetic criterion and, therefore, is minimized first without considering any other criteria. Thereby, an embedding of the input graph is computed. For non-planar graphs crossings are replaced by dummy nodes with degree 4 . The planarization together with its embedding is called topology. Generally, the topology is fixed throughout the remaining steps of the algorithm. In the second step, the shape of the final layout is optimized with respect to the fixed topology. The shape is the assignment of bends to edges (and the angles of these bends). Usually, the goal of this step is to minimize the number of bends. Finally, in the step metrics, the edge lengths, the node dimensions, and the node positions are determined. 
Our approach to compute column-based graph layouts is based on the TSMframework. This lets us split the problem of generating a graph layout into smaller subproblems and either solve them optimally or apply heuristics if they are $\mathcal{N} \mathcal{P}$-hard. In the topology step we minimize the number of crossing while enforcing that all edges are directed upwards. The result of this step is a so-called upward planar representation which prescribes the left-to-right order of the edges incident to each node. During the shape computation - the second step - we build upon this upward-planar representation in order to compute a so-called column assignment of the nodes and edges, i.e., we divide the plane into disjoint columns of uniform width and assign each node and each edge to a column. The column assignment induces four different properties - namely local symmetry, orthogonal edges, at most 4 bends per edge, and the port distribution. In the last step of the topology-shape-metrics-framework we only rely on the column assignment computed in the second step and do not respect the topology computed in the first step. In this step we compute the final coordinates of boxes and edge bends. Thereby, we minimize the total edge length and enforce correct box dimensions, minimum spacing constraints as well as alignment of predecessors.

Our approach has one important difference in comparison to the common usage of the topology-shape-metrics-framework. We do not fix the topology computed in the first step. We found out that during the shape and metrics phase minor changes to the topology can improve the æesthetics of the final layout. Thereby, we assign higher significance to bend and total edge length minimization than in usual applications of this framework. The remaining part of this section is structured along the topology-shape-metrics framework.

\subsection{Topology}

In this section we deal with the first step of the TSM-framework. Before we come to the main part of the topology-step, we conduct a preliminary step in which the input graph $G=(V, A)$ is transformed into an $s$-t-graph $\widehat{G}=(\widehat{V}, \widehat{A})$. This is done by adding a super source $\hat{s}$ and a super sink $\hat{t}$ to $G$ and connecting them with all of its sources and sinks, respectively. In the end, when converting the layout of $\widehat{G}$ to $G$, we simply omit $\hat{s}$ and $\hat{t}$ as well as all their incident edges.

We can now assume that the input is an $s$-t-graph $\widehat{G}$. We denote the unique source and sink by $\hat{s}$ and $\hat{t}$, respectively. Note that $\widehat{G}$ may be non-planar. We seek to minimize the number of crossings for the upward embedding. Since upward crossing minimization is $\mathcal{N} \mathcal{P}$-complete 8, we build on an algorithmic approach called "layer-free upward crossing minimization" by Chimani et al. 3]. The result of this algorithm is a so-called upward planar representation. An upward planar representation $\mathcal{U}$ of a DAG $G$ is an upward planar graph $\mathcal{U}=\left(V_{\mathcal{U}}, A_{\mathcal{U}}\right)$, in which edge crossings are replaced by crossing dummies, together with an upward planar embedding $\Gamma$. The arcs $a \in A$ are either elements of $A_{\mathcal{U}}$ as well or correspond to a path $p$ in $\mathcal{U}$. The inner nodes of such a path $p$ are crossing dummies, whereas the source and target node of $p$ correspond to the source and target of $a$, respectively.

In order to avoid one of the shortcomings of the Sugiyama framework Chimani et al. developed an upward crossing minimization method that works without a 
prescribed layer assignment of the nodes. We use their technique to minimize the number of crossings. Their approach is based upon two steps: (i) Delete edges until the remaining graph is upward planar and feasible, i.e., the deleted edges can be reinserted such that the resulting drawing is an upward drawing. (ii) Reinsert the deleted edges one by one. For each edge insertion at first a crossing-minimal reinsertion is conducted. However, a crossing-minimal reinsertion of an edge can prohibit the reinsertion of the remaining edges in a planar way. In this case, the reinsertion is undone and the edge is reinserted using a simple heuristic. Note that when reinserting edges, we do not count the crossings with edges incident to $\hat{s}$ or $\hat{t}$ since these edges are only used for technical reasons.

\subsection{Shape}

In this section we explain the second step of the topology-shape-metrics framework, i.e., how to compute the shape of a layout if an upward planar representation $\mathcal{U}$ of $\widehat{G}$ is given. In this context shape describes the number of bends on each edge and their bend directions.

The algorithm we present takes up the idea of Biedl and Kant on how to draw a graph with few bends in linear time 2] and adapts it to orthogonal upward drawings with at most four bends per edge. This approach leads to columnbased drawings. Since there are at most four bends per edge and ports are at the bottom and the top of the source and target, respectively, an edge can have at most three vertical segments. The column of the first (last) vertical segment is already determined by the column assigned to the source (target). The position of the middle vertical segment is determined by assigning it to some column. The result is a column assignments.

Definition 1 (Column Assignment). A column assignment of a DAG $G=$ $(V, A)$ is a mapping col: $V \cup A \rightarrow \mathbb{Z}$ which assigns each node $v_{i} \in V$ and each edge $e_{j} \in A$ to a column $\operatorname{col}\left(v_{i}\right)$ and $\operatorname{col}\left(e_{j}\right)$, respectively.

Note that a column assignment already induces the shape, i.e., which edges contain bends in which direction. In the metrics steps we will realize the column assignment, i.e., we compute a layout that respects the column assignment.

Definition 2 (Realization of a Column Assignment). Let $L$ be a valid layout of a $D A G G=(V, A)$, i.e., $L$ satisfies the local symmetry and four bends per edge constraints. Layout $L$ is a realization of a column assignment col, if we can divide the plane into disjoint columns of uniform width and number the columns from left to right such that each node $v_{i} \in V$ and each vertical edge $e_{j} \in A$ is positioned within column $\operatorname{col}\left(v_{i}\right)$ and $\operatorname{col}\left(e_{j}\right)$, respectively.

The algorithm that computes the column assignment works as follows: The nodes of $\widehat{G}$ are treated according to the $s$-t-order. Thus, we start with the super source $\hat{s}$ and assign it to column 0. Afterwards, we assign the outgoing edges of $\hat{s}$ according to the left to right order prescribed by $\mathcal{U}$ such that they are evenly distributed to the left and right of column 0 . 
The invariant of our algorithm is that all incoming edges of $v_{i}$ are already assigned to columns, when treating $v_{i}$ itself. Then we assign $v_{i}$ to column $m-$ the column of the median incoming edge - and assign the outgoing edges of $v_{i}$ to columns according to their left-to-right order induced by $\mathcal{U}$. However, the columns to the left and right of $m$ are possibly already occupied by other edges. Therefore, we shift all columns left (right) of $m$ to the left (right) such that there are out-degree $\left(v_{i}\right)-1$ empty columns which can be assigned with the outgoing edges of $v_{i}$ according to the left to right order prescribed by the upward planar representation $\mathcal{U}$. We now prove that the column assignment computed by our algorithm is realizable.

Theorem 1. There exists an algorithm that computes a realizable column assignment for a given $D A G \widehat{G}=(\widehat{V}, \widehat{A})$ in $\mathcal{O}(|\widehat{A}|)$ time and space.

Proof. A layout of $\widehat{G}$ can be constructed inductively during the execution of our shape algorithm. Since the $x$-coordinates are fixed by the column assignment, we only need to deal with the $y$-coordinates.

Initially, we set the $y$-coordinate of $\hat{s}$ to 0 and draw the first vertical and horizontal segments belonging to the outgoing edges of $\hat{s}$ as high as possible. When treating $v_{i}$ we draw the middle vertical segments of all incoming edges of $v_{i}$ such that they reach farther down than any other edge or box in the intermediate layout. Then we append the second horizontal segments of these edges and position the box $v_{i}$. Afterwards, we draw the first vertical and horizontal segment of the outgoing edges of $v_{i}$ as high as possible. Thus, no box can be intersected by an edge, and hence, this approach always yields a valid layout.

Since we assign nodes and edges to columns, each edge can contain at most two horizontal edge segments - one spanning from the source's column to the edge's column and one spanning from the edge's column to the target's column. Thus, there are at most four bends per edge. Furthermore, the shape algorithm positions the nodes and edges such that incoming and outgoing edges of each node $v_{i}$ are symmetrically distributed to the columns left and right of the column assigned to $v_{i}$. Thus, the layouts fulfil the local symmetry property.

The algorithm can be implemented straightforwardly to run in $\mathcal{O}(|\widehat{A}|)$.

For an upward planar representation without crossings this can be realized in a planar way.

Lemma 1. The realizations of the column assignments of an upward planar representation $\mathcal{U}$ that contains no crossing dummies are upward planar layouts.

During the construction of the column assignment, we only respect the left-toright ordering of the outgoing edges at each node of $\widehat{G}$ which is prescribed by $\mathcal{U}$. We do not consider the crossing dummies in $\mathcal{U}$. Thereby, we relax the topology that we computed in the first step. In particular, the column assignments computed by the shape algorithm can be realized in layouts that have different topologies.

Because of the relaxation of the topology, the final layout can have a higher number of crossings than the topology computed in the first step. However, this 
allows us to exploit the relaxation when minimizing the vertical edge length in the metrics step in order to gain more compact layouts. Thus, by relaxing the topology, we increase the significance of bend minimization and total edge length minimization in comparison to crossing number minimization. We do not focus on minimizing crossings since it has been established that crossing, where edges cross at large angles (in this case right angle crossings), are only a minor obstacle for humans to understand the relationship between nodes in graphs [9].

\subsection{Metrics}

In the last phase of the approach, we compute the final coordinates of boxes and edges and, thereby, minimize the total edge length. The topology that we originally computed in the first step has no influence on this step. Instead, we only rely on the column assignment computed by the shape algorithm.

The metrics step consists mainly of two substeps. First, we focus on minimizing the vertical edge length. Since we can show that this problem is $\mathcal{N} \mathcal{P}$ complete, we suggest a simple heuristic in order to solve it. Afterwards, we minimize the horizontal edge length. Note all operations are performed with respect to these columns so that the final result is a column-based layout.

Vertical Edge Length Minimization. We show that minimizing the total vertical edge length is $\mathcal{N} \mathcal{P}$-complete.

Theorem 2. Finding a realization of a column assignment with minimum total vertical edge length is $\mathcal{N} \mathcal{P}$-complete.

Since this problem is $\mathcal{N} \mathcal{P}$-complete we employ a greedy algorithm to minimize the vertical edge length. Recall that we want for each node to have as many predecessors as possible vertically aligned at their bottom. We integrate this requirement into our vertical edge length minimization approach. First, our greedy algorithm determines groups of such predecessors for each node. Second, we compute a reverse topological order for those groups and finally, we position the groups according to this order so that we minimize the vertical edge length.

We call these groups of nodes feasible if there is no directed path between two of its nodes. We call a node $n$ feasible with respect to a feasible group $g$ if, after adding $n$ to $g$, the group remains feasible and the node $n$ has not been assigned to a group yet.

We compute these feasible groups greedily until all nodes have been assigned to a group: Select a node $v$ that is not yet grouped and create a new group $g_{i}$ containing $v$. Add iteratively all feasible nodes from the set of the predecessors of $v$ 's successors to $g_{i}$. Consider then the predecessors of the successors of the newly added nodes and add the feasible nodes among them iteratively to $g_{i}$. Repeat this step until in one iteration no new nodes are added to $g_{i}$.

After computing the grouping, we compute an order of the groups in the second step. Since we draw the layout bottom-up in the third step, when positioning a group $g_{i}$ all successors of nodes in $g_{i}$ are already positioned. Thus, the order we compute needs to be a reverse topological order. 

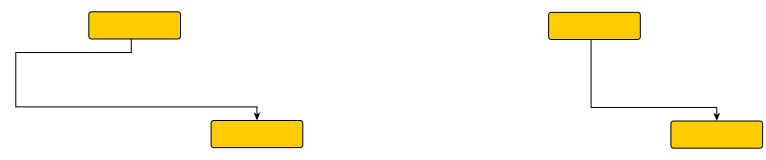

Fig. 2. An edge that is a bow (left) and the edge after bow reduction (right)

In the last step of the greedy approach the actual coordinate assignment takes place. We treat the groups according to the order computed in the second step. The $y$-coordinates of the ports and the bends of an edge are computed in two parts. When the target of an edge is positioned, we compute the $y$-coordinates of the target port and the first two bends. The first bend is in the target's column, whereas the other one is in the column assigned to the edge. The two last bends and the source port are computed, when its source is positioned.

At first, we compute the smallest possible $y$-coordinate $\hat{y}$ for the lower boundary of the nodes in $g_{i}$ by setting it to the maximum of the smallest possible $y$-coordinate for each node $v_{j} \in g_{i}$. When computing this $y$-coordinate for a node $v_{j} \in g_{i}$ we need to consider that we have not yet routed the second part of the edges whose targets are in $g_{i}$. For this, we need to compute the smallest possible $y$-coordinate for the horizontal edge segment of the edges we still need to draw. In particular, we need to find the smallest possible $y$-coordinate such that all minimum spacing constraints are respected. This can be done straightforwardly.

After determining $\hat{y}$, we position the boxes in $g_{i}$ such that their lower boundary is aligned at $\hat{y}$ and draw the second part of the outgoing edges. We draw the horizontal segments as high as possible. Afterwards, we draw the incoming edges as low as possible. To this end, we process the nodes $v_{j} \in g_{i}$ in an arbitrary ordering and draw their incoming edges such that all spacing constraints hold.

The vertical edge length minimization heuristic can be implemented to run in $\mathcal{O}((|\widehat{V}|+b) \cdot|\widehat{A}|)$ time and $\mathcal{O}\left(|\widehat{V}|^{2}\right)$ space, where $b$ is the number of columns computed by the shape algorithm.

Horizontal Edge Length Minimization. There are two steps we conduct for the horizontal edge length minimization. First, we remove some unnecessary structures, called bows, that are caused by the shape algorithm; see Figure 2 . This can be done straightforwardly and requires only linear time.

In the second step, we employ a heuristical approach to minimize the width of the whole layout. Due to the shifting in each iteration of the shape algorithm, we introduce new columns to the shape. Some of them are necessary while others will be partly or even completely empty in the final layout. Thus, the final layout is wider than actually necessary. In Figure 3 we depict a layout that can be compacted by two columns. Our approach to width compaction is column based, i.e., the resulting layout again consists of disjoint columns.

We perform width compaction along so-called compaction paths. A compaction path is an axis-parallel $y$-monotone path passing through a layout from the top to the bottom. It may only cut horizontal edge segments. Furthermore, its vertical 


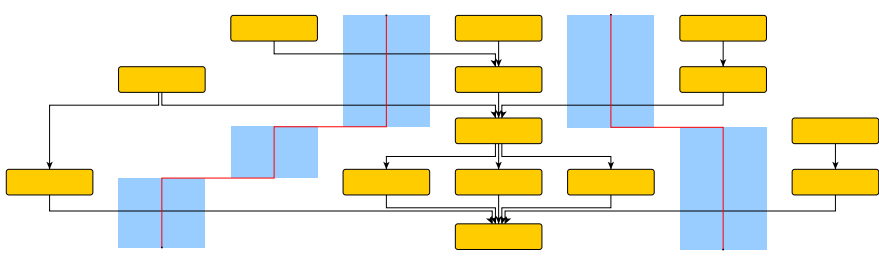

Fig. 3. A layout that can be compacted by two columns and two compaction paths

segments need to run within columns such that no vertical edge segment is allowed to run through the same part of these columns. Each compaction along a compaction path will reduce the number of columns by one.

A seemingly obvious approach to compute a maximum set of compaction paths would be by using a flow algorithm. However, there are examples of compaction paths where each path by itself is valid, but compacting two paths at the same time yields a layout where an edge-edge spacing constraint is violated. Thus, we need a different approach to compute a cardinal-maximal valid set of compaction paths. We call a set of compaction paths valid if, after compacting a layout along all its compaction paths, the result is a valid layout. In particular this means that after the compaction no spacing constraint can be violated.

Our approach works as follow: First, it greedily selects from right-to-left a maximum set of disjoint valid compaction paths. Note that although no two paths may overlap, crossings are allowed. Hence, in the second step of the algorithm all crossings between the paths are removed. Since now the paths can be ordered from right to left we iteratively compact along the paths in this order. This is summarized in the following theorem. Note that we show here only that the number of paths remain unchanged after removing the crossings. The full proof of the theorem can be found in the appendix.

Theorem 3. There exists an algorithm that determines a maximum set of compaction paths and minimizes a given layout of $D A G \widehat{G}=(\widehat{V}, \widehat{A})$ in $\mathcal{O}(|\widehat{A}| \cdot b$. $(\log (|\widehat{V}|)+b))$ time and $\mathcal{O}\left(|\widehat{A}| \cdot b^{2}\right)$ space, where $b$ is the number of columns computed by the shape algorithm.

Proof. Here we show that the number of paths in the maximal set remains unchanged after we remove all crossings.

If two compaction paths $p_{i}$ and $p_{j}$ cross each other, we swap the suffixes of the paths that start at the crossing. Afterwards, $p_{i}$ and $p_{j}$ touch each other but they do not cross anymore. Furthermore, we cannot introduce new crossings with this approach. Thus, the overall number of crossings decreases by one in each step while the total number of compaction paths remains unchanged.

In total, our algorithm computes a column-based layout for a given $s$ - $t$ graph $\widehat{G}=(\widehat{V}, \widehat{A})$ in $\mathcal{O}\left(|\widehat{A}|^{2} \cdot(|\widehat{V}|+b)\right)$ time and $\mathcal{O}\left(|\widehat{V}|^{2}\right)$ space, where $b$ is the number of columns computed by the shape algorithm. 


\section{Evaluation}

The drawing style used here stems from a particular application for drawings of so-called argument maps. Argument maps present the arguments given in a debate or a book together with two binary relations among them, i.e., support and attack. They originate from argumentation theory but are used in many more fields like philosophy and politics. For detailed background information about argument maps we refer the reader to [1]. For layouts of argument maps we add additional constraints which we did not describe yet, namely free sources and sinks. Thereby, we denote the property that above a source and below a sink no other box is positioned. We can enforce this constraint by not removing the edges incident to $\hat{s}$ and $\hat{t}$ before the width compaction. Then, these avoid that another box is shifted above a source or below a sink.

As already mentioned in Section 2.1 we randomize the computation of the topology. We do this at two points: (i) for the construction of the feasible upward planar subgraph and (ii) we randomize the order in which the deleted edges are reinserted. These two steps are performed ten times, i.e., we execute the computation of a feasible upward planar subgraph 100 times in total. Over all runs we take the crossing minimal upward planar representation as result.

The basis of this evaluation is a set of 51 argument maps differing in size, purpose of usage and experience of the creator (uploaded to http://www.grapharchive.org). For a more detailed analysis of these input graphs we refer to [6]. First, we present statistics about measurable quality criteria, and then discuss the æsthetic qualities of these layouts.

Statistics. We start by analyzing the number of crossings in the final layout as well as in the upward planar representation $\mathcal{U}$. Although it is theoretically possible that the final layout contains less crossings than $\mathcal{U}$, for all instances there are at least as many crossing in the final layout as in the upward planar representation $\mathcal{U}$. However, if $\mathcal{U}$ is free of crossings, then the final layout is planar as well (compare to Lemma 1). On average there are $69 \%$ more crossings in the final layouts than in the upward planar representations.

Due to the shape algorithm there can be at most four bends per edge. Except of three bend-free layouts, the average number of bends per edge lies between 0.4 and 1.5. Only 13 of 51 layouts have edges with four bends. Taking the average over all layouts, we have 1.06 bends per edge.

Finally, we turn towards the runtime analysis. The maximum runtime for the 51 input instances is $25 \mathrm{~s}$ for an instance having 134 nodes and 158 edges, whereas the average is $0.77 \mathrm{~s}$. The average runtime is strongly influenced by the few large instances. For instances with at most 30 nodes, the runtime on average is $0.06 \mathrm{~s}$ while the maximum runtime is $0.52 \mathrm{~s}$.

It is interesting to note the contribution of the three phases topology, shape and metrics to the overall runtime. Shape and metrics make only insignificant contributions of $<0.01 \%$ and $0.4 \%$, respectively, whereas topology needs $99.6 \%$ of the overall runtime. Thus, the topology step is the bottleneck and therefore, 


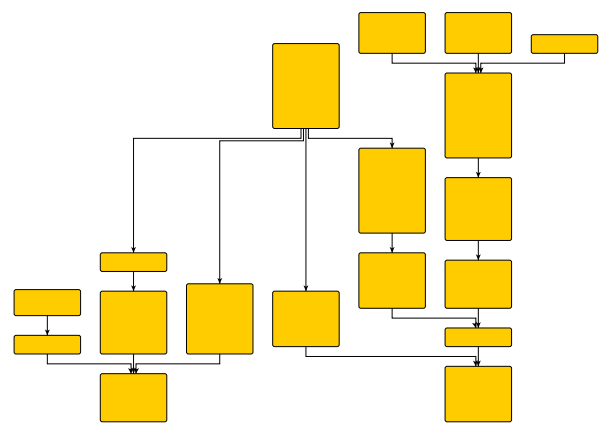

Fig. 4. Instance D-2.5

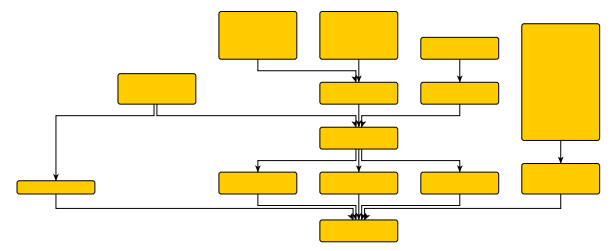

Fig. 5. Instance D-5.3

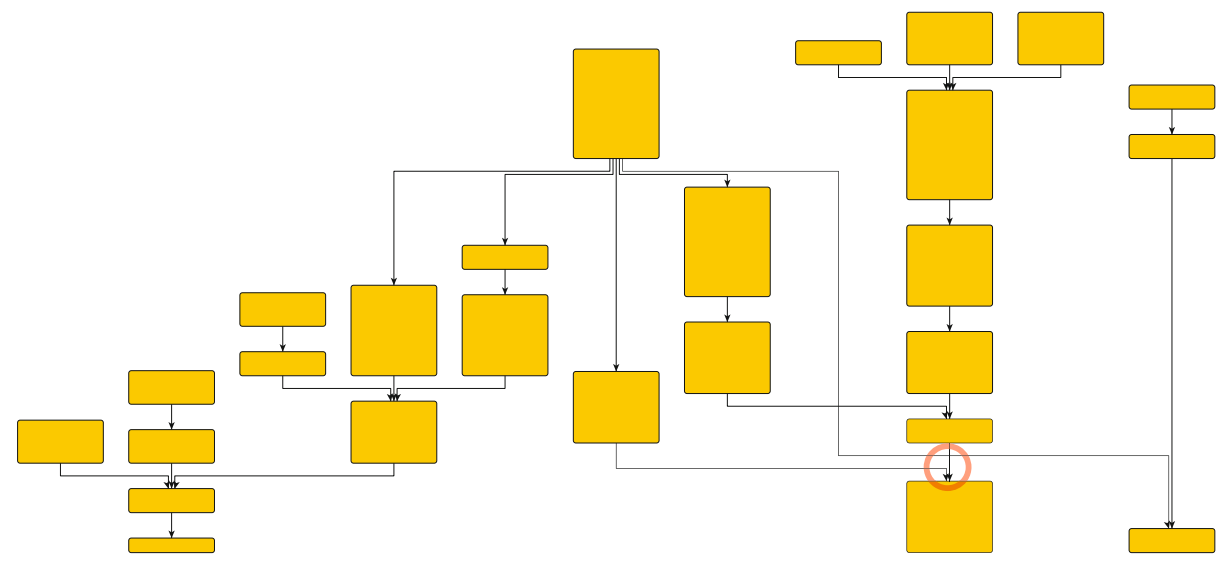

Fig. 6. Instance D-2.7. Circle added to highlight effect of different edge-edge spacing

to decrease the overall running time we suggest decreasing the number of randomized runs of the topology step.

Case Studies. In this section we present three layouts that are created by the algorithms described in Section 2. Using these layouts as examples we discuss the benefits and the disadvantages of these algorithms. Figure 4 shows a layout for which we have no suggestions for improvement. The layout is free of crossings, the number of bends are close to the minimum, it is compact and locally symmetric. Beside the hard facts, it is well structured and gives a good impression of the internal structure. The other two layouts - though they are good looking in general - have minor drawbacks which we discuss in the following.

In Figure 5 on the left side there is a constellation that is similar to a bow which we treated in Section 2.3. However, this time we have a bow containing a box. We could expand this concept to paths in $\widehat{G}$ that contain only nodes whose in-degree and out-degree equals one. However, the scenario depicted in Figure 5 only occurs 5 times in the 51 input instances. Therefore, we omit its treatment. 
In Figure 6 there is a set of boxes on the right side of the layout that is positioned quite high. It seems as it might be possible to move these boxes downwards. However, these boxes are aligned with boxes that are some columns apart, i.e., they need to be positioned that high. One could reconsider the drawing style, such that alignment of boxes is only required if the number of columns between the boxes is small. Note that we can easily integrate such a modification by adapting the computation of the groups in Section 2.3. Besides this drawback, the example gives a good impression of the edge bundling enabled by the minimum edge-edge spacings. The effect the two different edge-edge spacing constraints have on the final layout can be seen inside the red circle. The two edges that have the same target have a small distance between them while the third horizontal edge-segment belonging to the third edge is placed at a greater distance to the other horizontal edge-segment.

Overall, we conclude that the computed layouts are of high quality from an æesthetic point of view as well as regarding the statistics.

\section{References}

1. Betz, G.: Theorie dialektischer Strukturen. Klostermann (2010)

2. Biedl, T., Kant, G.: A Better Heuristic for Orthogonal Graph Drawings. In: van Leeuwen, J. (ed.) ESA 1994. LNCS, vol. 855, pp. 24-35. Springer, Heidelberg (1994)

3. Chimani, M., Gutwenger, C., Mutzel, P., Wong, H.M.: Layer-free upward crossing minimization. Journal of Experimental Algorithmics 15 (2010)

4. Chimani, M., Gutwenger, C., Mutzel, P., Wong, H.M.: Upward planarization layout. Journal of Graph Algorithms and Applications 15(1), 127-155 (2011)

5. Di Battista, G., Didimo, W., Patrignani, M., Pizzonia, M.: Orthogonal and Quasiupward Drawings with Vertices of Prescribed Size. In: Kratochvíl, J. (ed.) GD 1999. LNCS, vol. 1731, pp. 297-310. Springer, Heidelberg (1999)

6. Doll, C.: Automatic Layout Generation for Argument Maps. Master's thesis, Karlsruhe Institute of Technology (February 2012)

7. Eades, P., Tamassia, R.: Algorithms for drawing graphs: An annotated bibliography. Tech. rep., Brown University, Providence, RI, USA (1988)

8. Garg, A., Tamassia, R.: On the computational complexity of upward and rectilinear planarity testing. SIAM Journal on Computing 31, 601-625 (2002)

9. Huang, W., Hong, S.H., Eades, P.: Effects of crossing angles. In: IEEE Pacific Visualization Symposium, PacificVIS 2008, pp. 41-46 (2008)

10. Sugiyama, K., Tagawa, S., Toda, M.: Methods for visual understanding of hierarchical system structures. IEEE Transactions on Systems, Man and Cybernetics 11(2), 109-125 (1981)

11. Tamassia, R.: On embedding a graph in the grid with the minimum number of bends. SIAM Journal on Computing 16, 421-444 (1987) 